\title{
Correspondence
}

\section{Comment on: Adrenal hypoplasia congenita in identical twins}

\section{To the Editor}

Follow up of the case report "Adrenal hypoplasia congenita in identical twins", published in Saudi Medical Journal in January 2019. ${ }^{1}$

The case was about a monozygotic twin brothers who presented at different ages with different presentations with confirmed genetic testing of DAX1 (NR0B1) mutation. Adrenal hypoplasia congenita is frequently associated with hypogonadotropic hypogonadism, the spectrum of presentation varies widely from pubertal failure to infertility.

The twin brothers were followed up in endocrine clinic every 3 to 4 months and their pubertal development was assessed in each visit, with the assessment of follicle-stimulating hormone (FSH), luteinizing hormone $(\mathrm{LH})$, and testosterone level every 6 months. Both of them proceeded smoothly in normal puberty.

Their last visit was on June 2020. Their growth parameter based on CDC growth chart:

Twin A: height $=147 \mathrm{~cm}$ ( $3^{\text {rd }}$ centile $)$, weight: $33 \mathrm{~kg}$ (below $3^{\text {rd }}$ centile), Pubertal staging: testes Tanner stage IV \& pubic hair Tanner stage III (Table 1).

Twin B: height $=147 \mathrm{~cm}$ ( $3^{\text {rd }}$ centile), weight: $43 \mathrm{~kg}$ (below $10^{\text {th }}$ centile), Pubertal staging: testes Tanner stage IV \& pubic hair Tanner stage III (Table 2).

Both of them proceeded smoothly in normal puberty.

In conclusion, our case study for the twin brothers with adrenal hypoplasia congenita, that both of them were having normal pubertal development.
Table 1 - Twin A’s laboratory results.

\begin{tabular}{lccc}
\hline Date & $\begin{array}{c}\text { LH } \\
(\mathbf{0 . 2 6 - 4 . 8} \\
\text { IU/L) }\end{array}$ & $\begin{array}{c}\text { FSH } \\
(\mathbf{0 . 3}-4.6 \\
\text { IU/L) }\end{array}$ & $\begin{array}{c}\text { Testosterone } \\
(\mathbf{0 . 1 7 - 2 . 4 3} \\
\text { nmol/L) }\end{array}$ \\
\hline May 2018 & 0.92 & 2.58 & 1.8 \\
December 2018 & 2.06 & 2.82 & 0.04 \\
February 2019 & 0.1 & 2.73 & 0.09 \\
September 2019 & 0.42 & 3.08 & 6.13 \\
June 2020 & 1.11 & 2.87 & 10.86 \\
\hline
\end{tabular}

Lh: luteinizing hormone, FSH: follicle-stimulating hormone

Table 2 - Twin B's laboratory results.

\begin{tabular}{lccc}
\hline Date & $\begin{array}{c}\text { LH } \\
(\mathbf{0 . 2 6 - 4 . 8} \\
\text { IU/L) }\end{array}$ & $\begin{array}{c}\text { FSH } \\
(\mathbf{0 . 3}-\mathbf{4 . 6} \text { IU/L) }\end{array}$ & $\begin{array}{c}\text { Testosterone } \\
(\mathbf{0 . 1 7}-\mathbf{2 . 4 3} \\
\mathbf{n m o l} / \mathrm{L})\end{array}$ \\
\hline May 2018 & 0.67 & 2.76 & 0.09 \\
December 2018 & 0.77 & 2.85 & 0.09 \\
February 2019 & 0.1 & 1.94 & 0.09 \\
September 2019 & 0.43 & 3.66 & 4.07 \\
June 2020 & 2.43 & 5.92 & 8.57 \\
\hline
\end{tabular}

Lh: luteinizing hormone, FSH: follicle-stimulating hormone

\section{Alia M. Al Amer \\ Kholud M. Al Rubaya \\ Unit of Pediatric Endocrinology and Diabetes \\ Maternity and Children Hospital \\ Dammam, Kingdom of Saudi Arabia}

\section{References}

1. Amer AM, Al Rubaya KM, Alzahrani AS. Adrenal hypolasia congenita in identical twins. Saudi Med J 2019; 40: 87-92. 